\title{
Curvature and Vibration Sensing Based on Core Diameter Mismatch Structures
}

\author{
Cindy S. Fernandes, Maria Thereza M. Rocco Giraldi, Marco J. de Sousa, João C. W. A. Costa, Member, IEEE, \\ Carlos Gouveia, Pedro Jorge, and Marcos A. R. Franco
}

\begin{abstract}
Core diameter mismatch structures are proposed and experimentally investigated for curvature and vibration sensing. Two configurations are suggested, one approach uses a structure formed by splicing an uncoated short section of multimode fiber between two standard single-mode fibers (SMFs) single-mode-multimode-single-mode (SMS), combined to a fiber optical mirror at its end, and the other approach uses a structure made by splicing a section of SMF between two multimode fibers (SMSMS). In the curvature analysis, the proposed SMS sensor generates the destructive interference patterns when it is bent, varying only the attenuation of the optical signal without wavelength shifts. The SMSMS vibration sensor proved to be suitable to monitor very low frequencies such as $0.1 \mathrm{~Hz}$. The configuration of the proposed sensors presents several interesting features, such as easy fabrication, low cost, high efficiency, and high sensitivity. These advantages make such sensors very useful in a wide range of applications, for instance, structural health monitoring.
\end{abstract}

Index Terms-Core diameter mismatch (CDM), optical curvature sensor, optical vibration sensor, single-modemultimode-single-mode (SMS), SMSMS.

\section{INTRODUCTION}

$\mathbf{R}$ ECENT advances and cost reductions in optical devices have stimulated the interest in optical fiber sensors applied to measure the physical and mechanical parameters, mainly because of potential applications in several fields, for instance, structural health monitoring (SHM) [1]-[3]. In this case, real-time measurements of strain, curvature, vibration,

Manuscript received January 14, 2016; revised March 28, 2016; accepted April 25, 2016. Date of publication June 6, 2016; date of current version August 9, 2016. This work was supported in part by the Vale S.A., in part by the National Council for Scientific and Technological Development, in part by the Coordination for the Improvement of Higher Education Personnel, in part by the Fundação de Amparo à Pesquisa do Estado do Rio de Janeiro, and in part by the Portugal Agency Fundação para a Ciência e a Tecnologia. The Associate Editor coordinating the review process was Dr. George Xiao.

C. S. Fernandes, M. J. de Sousa, and J. C. W. A. Costa are with the Federal University of Pará, Belém 66075-110, Brazil (e-mail: cindystella@ufpa.br; marcojsousa@ufpa.br; jweyl@ufpa.br).

M. T. M. R. Giraldi is with the Military Institute of Engineering, Rio de Janeiro 22290-270, Brazil (e-mail: mtmrocco@ime.eb.br).

C. Gouveia is with the Federal University of Campina Grande, Campina Grande 58109-970, Brazil, and also with the Instituto de Engenharia de Sistemas e Computadores, Tecnologia e Ciência, Porto 4169-007, Portugal (e-mail: icarlos07@gmail.com).

P. Jorge is with the Instituto de Engenharia de Sistemas e Computadores, Tecnologia e Ciência, Porto 4169-007, Portugal (e-mail: pjorge@inescporto.pt).

M. A. R. Franco is with the Institute of Advanced Studies, São José dos Campos 12228-001, Brazil (e-mail: marcos@ieav.cta.br).

Color versions of one or more of the figures in this paper are available online at http://ieeexplore.ieee.org.

Digital Object Identifier 10.1109/TIM.2016.2571378 and other parameters are crucial for identification, localization, and quantification of structural damages, and also improve the maintenance and safety of the monitored structures [4]-[7].

Among the aforesaid parameters, the curvature and vibration measurements are important to monitor due to their relationship with the dynamic responses of engineering structures [8]-[11]. Curvature sensors appear as an alternative to strain gauges for SHM applications [12], for example, in the evaluation of a curvature deflection. The usage of electrical or optical strain gauges to measure the mechanical strain on the structural surface becomes too expensive when small deflections must be measured as, in this case, a very high resolution is required [13]-[15]. Curvature measurements can be performed at any location across the cross section and provide an indication of any change in the stiffness of a structure subject to bending [9], [10], [13]-[15]. Therefore, curvature sensors are used with the advantage that the curvature deflection does not depend on its structural thickness.

In turn, vibration sensors are essential for the structural assessment, needful in the early detection of anomalies to avoid malfunction or collapses [4], [11]. However, vibration measurements demand acquisition schemes with higher sampling rate than the normally used by optical instruments to acquire quasi-static signals [16], [17]. Moreover, to detect the frequencies ranging from $0.1-10 \mathrm{~Hz}$, electronic seismic accelerometers with high sensitivity (less than one thousandth of $\mathrm{g}$ or $0.01 \mathrm{~m} / \mathrm{s}^{2}$ ) are often used [18].

There are several devices and widespread techniques to perform optical sensing, such as fiber Bragg grating (FBG) [19], [20], long period grating [21], [22], and Fabry-Perot interferometer [23]. In particular, the intrinsic sensitivity of FBG makes this technology a satisfactory candidate, commonly applied in structural sensing. An FBG sensor is an optical reflection filter that changes its spectral response according to the temperature and strain variations [24]. Thereby, to acquire measurements from an FBG sensor, a specific spectrum analysis is necessary, which is relatively complex and demands expensive equipment but also allows multiplexing with several FBGs in series in the same optical fiber [24], [25]. As strain sensors, the FBGs can be used to monitor vibrations in a wide range of frequencies, because the light readily responds to any geometric change of the FBG structure with delays given in function of the light speed [26], [27]. Unfortunately, due to the temperature sensibility of FBG, the compensation techniques are required. Usually, the FBG strain sensor 
is applied together with an FBG temperature sensor [28]. The FBGs are also used in optical accelerometers, where a mechanical mounting translates acceleration to displacement or strain which is measured by an FBG [16], [29], [30]. The resistance and limited deformation capacity of optical fiber deteriorate the performance of optical accelerometer when compared with electronic ones. Optical accelerometers have not yet fully achieved the sensitivity and frequency response of electronic seismic accelerometers at frequencies less than $1 \mathrm{~Hz}$ [31], [32].

Other approaches for curvature sensors use sensitized optical fibers to induce signal loss, which can be easily measured. In [14], a step-index multimode optical fiber is subjected to transversal cuts into its core. High sensitivity is achieved with hundreds of cuts spread along $75 \mathrm{~mm}$ of fiber. In [33], a plastic optical fiber (POF) is sensitized using a similar approach as [14], but achieving shorter sensors of $\approx 15 \mathrm{~mm}$. Both sensors [14], [33] are inexpensive and operate diminishing the transmitted optical power. However, they also result in relatively large sensors, which restricts the universe of structures able to be monitored. The loss of the chosen multimode optical fibers, particularly the POF, also obligates the interrogation and measurement apparatus to be placed meters away from the sensor, which is not desirable in structural monitoring applications. In [34], a fiber core diameter mismatch (CDM) is made by inserting $4 \mathrm{~mm}$ of thin-core fiber (TCF) in the singlemode fiber (SMF). The resulting sensor has high sensitivity to curvature, is compact and can be placed far away (several kilometers) from the interrogation and measurement setup. However, the TCF sensor is interferometric in nature, demonstrating considerable temperature dependence and demanding interrogation apparatus similar to those used to FBG sensors.

In the last years, single-mode-multimode-singlemode (SMS) fibers devices have been proposed as optical sensors due to their low cost and simple fabrication [35]-[38]. The SMS optical sensor is formed by splicing a section of multimode fiber (MMF) between two SMFs [39]-[42]. An SMS curvature sensor that uses the CDM technique [43] is proposed. Different from some multimode interference (MMI) structures [44], [45], the SMS structure reported here generates destructive interference patterns, which does not vary the optical power with the wavelength, only introducing a loss in the optical signal [46]. In this regard, the attenuation of the optical signal will vary when the fiber is bent allowing to interrogate the sensor. Based on the aforementioned principle, this paper also proposes an in-fiber interferometer vibration sensor consisting of an SMF input fiber, a section of uncoated SMF spliced between two MMFs, and an SMF output fiber, i.e., an SMSMS structure. This optical sensor has been reported for refractive index and temperature measurements [47]. The feasibility of such structure is extended to measure the vibrations at very low frequencies, less than $1 \mathrm{~Hz}$.

In this paper, two configurations are proposed: 1) a configuration based on the SMS fiber structure and an Optical time domain reflectometer (OTDR)-based interrogation technique for a remote curvature sensing and 2) a configuration based on the SMSMS fiber structure and an acquisition

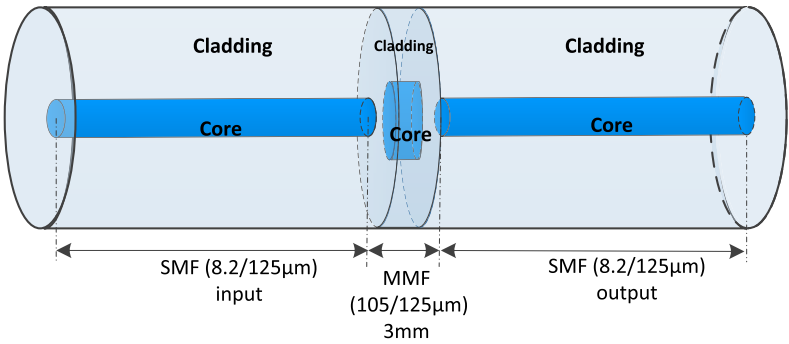

Fig. 1. Configuration of the proposed SMS curvature sensor.

system based on Arduino for vibration sensing. This paper improves the possibility of such fibers structures for curvature measurements and vibration detection. The aim is to analyze the sensitivity response of a remote curvature sensor when it is bent and a vibration sensor to detect very low frequencies. The proposed experimental schemes use information from the OTDR reflection peak variation to evaluate the SMS sensor response and the data acquired from an acquisition system based on Arduino to analyze the vibrations measured by the SMSMS sensor.

This paper is organized as follows. Section II presents the fabrication and the sensing principle of the SMS structure as a remote curvature sensor and the SMSMS device as a vibration sensor. In Section III, the experimental results of the SMS sensor through displacement variations and temperature influence, and the SMSMS sensor via frequency identification tests are described. Experimental results of the SMS sensor and numerical simulations based on a 3-D beam propagation method (BPM) are discussed and compared in Section IV. Finally, in Section V, the conclusions are exposed due to the utilization of the proposed curvature and vibration sensors.

\section{SEnSOR Fabrication}

The remote curvature sensor is based on an SMS structure, which is schematically shown in Fig. 1. The MMF section in the sensor, due to the large CDM, acts as a core-cladding coupling mechanism [48]. Multiple modes will be excited in the MMF section when the light comes in from the SMF. Due to its short length, the MMF section generates destructive interference patterns. The MMF couples part of the light traveling along the core of the first SMF to the cladding of the second one, and this coupling induces a loss of power in the transmitted signal traveling in the core. This coupling is not selective in terms of wavelength, i.e., it is wavelength independent. Thus, the proposed device does not act as a common MMI and merely introduces a loss in the optical signal, which makes it attractive for several applications. When the sensing structure is bent, the coupling coefficient of the structure is changed, varying the attenuation in the transmitted signal.

The SMS device is fabricated by splicing a short section of uncoated step-index MMF (FG105-LCA from Thorlabs, Inc.), with a length of $3 \mathrm{~mm}$, between the input and output standard SMFs (SMF-28 from Corning, Inc.). Regarding the short section length of the MMF fiber, $3 \mathrm{~mm}$ is used due to its high sensitivity, as demonstrated in [43]. Two SMS sensors, 


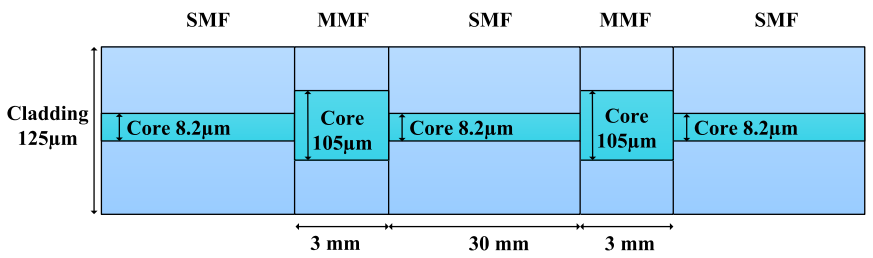

Fig. 2. Configuration of the proposed SMSMS vibration sensor.

under the aforementioned parameters, are developed and later subjected to curvature tests. First, a piece of MMF is spliced to a section of SMF, and then the MMF is cleaved with a length of $3 \mathrm{~mm}$, which is measured by a ruler. Finally, another section of SMF is spliced to the cleaved MMF.

Although the manufacturing process of the sensor head is simple, it is not very precise, so the heads are not exactly equal, which tightly affect their sensitivity. However, a solution for this problem is to improve the fabrication process of the sensors by using a precision linear stage to measure and cleave the fiber. The reported sensors are fabricated with the core/cladding diameters of the $\mathrm{SMF} \approx 8.2 / 125 \mu \mathrm{m}$; meanwhile for the step-index MMF section, the core/cladding diameters are $105 / 125 \mu \mathrm{m}$.

To improve the sensitivity of the SMS structure and extend the viability of such structure to measure vibrations, an in-fiber interferometer vibration sensor, consisting of an input SMF, a section of uncoated SMF spliced between two MMFs and an output SMF, is developed. This structure, therefore, is called the SMSMS structure. The two MMFs act as mode couplers to split and recombine light due to the CDM technique [43].

The SMSMS vibration sensor, as shown in Fig. 2, is fabricated by splicing a $30 \mathrm{~mm}$ long uncoated standard SMF between two MMFs and the MMFs are spliced to two SMFs at the input and output of the device, respectively. The core/cladding diameters of the SMF and MMF are 8.2/125 $\mu \mathrm{m}$ and $105 / 125 \mu \mathrm{m}$, respectively. The MMF length, with $3 \mathrm{~mm}$ each, has an impact on the interference spectrum, because it determines the mode-coupling coefficient when the light in the MMF couples into the uncoated SMF.

As the source of light, a laser source operating at the wavelength of $1550 \mathrm{~nm}$ with $-5 \mathrm{dBm}$ of optical power is used. The light is launched into the first MMF through the input SMF, and higher order modes are excited due to CDM. When light reaches the uncoated SMF, a portion of the light enters its core, while the remaining portion is coupled into the cladding of this fiber. These modes reach the second MMF and propagate through it. The core and cladding modes with different propagation constants interfere with each other. Then, the light is recoupled into the output SMF.

The optical power of SMS and SMSMS sensors presents a loss caused by splicing for both structures. To confirm these losses, an optical laser source operating at the range of $1550 \mathrm{~nm}$ with $-4 \mathrm{dBm}$ is applied to an SMS and to an SMSMS structure. The measurements are performed using an optical power meter device. The measurements results describes a power loss of $\approx 11 \mathrm{~dB}$ to the SMS sensor and $\approx 15 \mathrm{~dB}$ to the SMSMS sensor. These initial losses of both sensors, showed on the optical power meter,

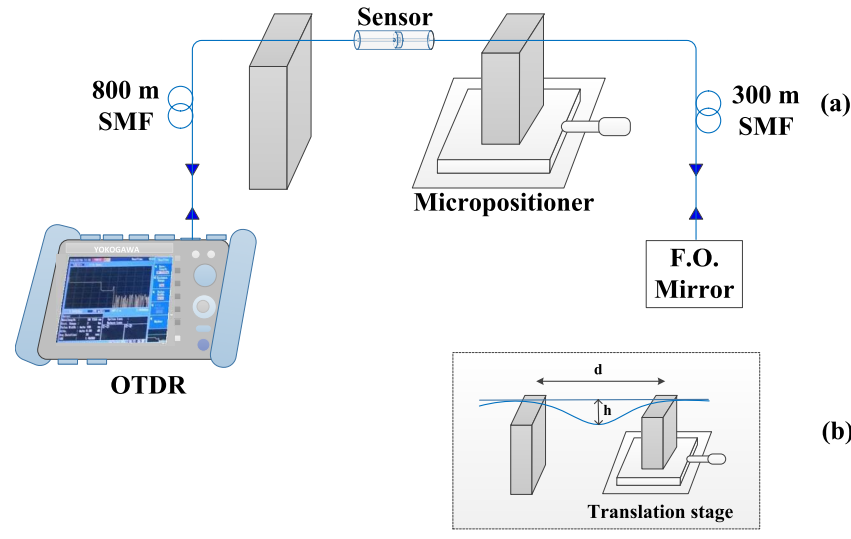

Fig. 3. (a) Experimental setup for remote curvature measurements using the OTDR. (b) Moveable stage with a translation stage (bend stage).

are obtained when the bent and vibration are not applied, i.e., the sensors have intrinsic losses due to the splicing process before any measurement being performed. Besides, when the experimental results are compared with the simulated ones, which do not present initial losses, the results are normalized and the difference between both is mitigated.

\section{EXPERIMENTAL ANALYSIS}

First, to ensure that the SMS device can work at different wavelengths, an optical beam from a broadband source operating at the range from 1500 to $1580 \mathrm{~nm}$ is applied on it. The measurements are performed using an optical spectrum analyzer. The results demonstrated that when the bent is applied, a loss is merely introduced in the signal power. The induced loss is independent of the wavelength.

Afterward, the SMS sensor is interrogated using an OTDR, as shown in Fig. 3(a). A commercial OTDR from YOKOGAWA, model AQ 1200 OTDR-Multi Field Tester with the operating wavelength of $1550 \mathrm{~nm}$, a pulsewidth of $100 \mathrm{~ns}$, and an average duration of $30 \mathrm{~s}$, is used. Remote sensing is obtained connecting $800 \mathrm{~m}$ of Corning SMF-28 fiber to the OTDR and $300 \mathrm{~m}$ of Corning SMF-28 fiber with a mirrored end splicing to the sensor output. The $25 \%$ optical fiber mirror is used to increase the reflected power of the transmitted signal, and consequently enhances the resolution due to the increment in the signal-to-noise ratio.

The curvature measurements are performed by straightly fixing the SMS sensor on two blocks distanced $22 \mathrm{~cm}$ by each other. One of the blocks is mounted on a translation stage to enable applying displacement and inducing a bending on the SMS fiber structure, as shown in Fig. 3(b). The measurements are performed with a displacement range from 0 to $600 \mu \mathrm{m}$, with an increment of $100 \mu \mathrm{m}$. If the SMS sensor is placed at a half distance between the two mounting blocks, then the fiber bend that is defined as the inverse of the bend radius $(1 / R)[49]$ is given by

$$
C=\frac{1}{R}=\frac{2 h}{h^{2}+(d / 2)^{2}}
$$

where $d=L_{0}-\Delta L, L_{0}$ is the initial distance between the two blocks, $\Delta L$ is the distance variation and $h$ is the 


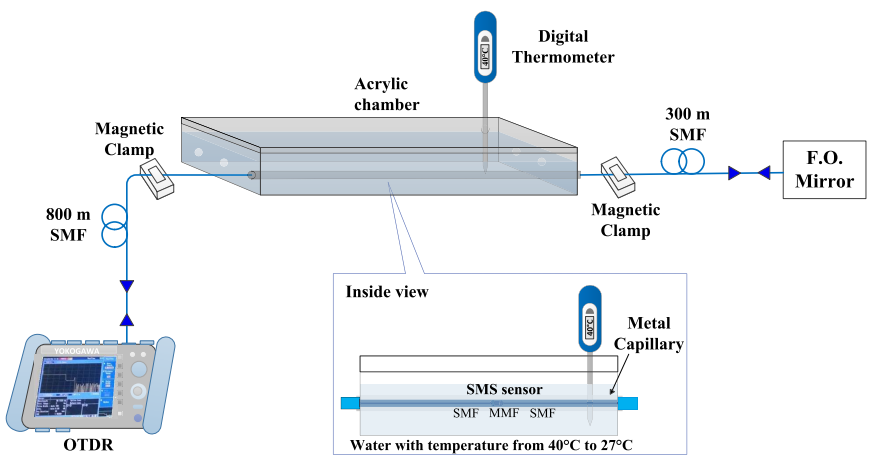

Fig. 4. Experimental setup for the temperature analysis.

bend deepness. An approximation of (1) is used in this paper as the displacement steps are of the order of some micrometer and the measurement of the bend size is, therefore, difficult. The bend radius as a function of $L_{0}$ and $d$ is achieved as follows:

$$
C=\frac{1}{R} \approx \frac{4}{L_{0}^{2}}\left(L_{0}^{2}-d^{2}\right)^{\frac{1}{2}} .
$$

When the light from the OTDR is injected into the straight fiber, light propagation in the MMF section is symmetrically distributed. For a bent MMF, the refractive index is no longer symmetrical along the fiber axis and part of the power is lost in the cladding modes of the fiber. The loss increases if the curvature is applied.

To analyze the influence of the temperature on the SMS sensor, a simple experiment is implemented. To perform the temperature measurements, a variation in the temperature in the range from $27{ }^{\circ} \mathrm{C}$ to $40{ }^{\circ} \mathrm{C}$ is applied, whose values are chosen due to the interest on environmental temperatures. As well as in the curvature measurements, this analysis used the wavelength of $1550 \mathrm{~nm}$ in the OTDR equipment and the results are based on a power difference. The experiment is developed in water as it presents higher temperature stability.

The experimental setup is the same represented in Fig. 3, but the response of the sensor to the applied temperature is investigated by introducing the sensor head into a metal capillary, which is within an acrylic chamber, as shown in Fig. 4. For this analysis, the fiber remained stretched and fixed in two points separated by $12 \mathrm{~cm}$, then the effects of swelling and shrinking of fiber become minimum according to theoretical calculations. Without bending, the temperature variation is the only parameter to be measured and it causes fiber dilatation. The water is heated in a hotplate and placed in the acrylic chamber, where the temperature is measured with a digital thermometer.

For the vibration sensing analysis, an experimental verification of the usefulness of the SMSMS structure as a sensor is carried out. The schematic of the experimental configuration is shown in Fig. 5, which comprises a laser source [Fig. 5(a.I)], a vibration exciter from Brüel \& Kjær, type 4809 [Fig. 5(a.II)], an optical Data Acquisition (DAQ) system based on an Arduino configuration [Fig. 5(a.III)], a reference electronic accelerometer, and the SMSMS sensor [Fig. 5(b)]. The frequency response of the SMSMS sensor is obtained using a

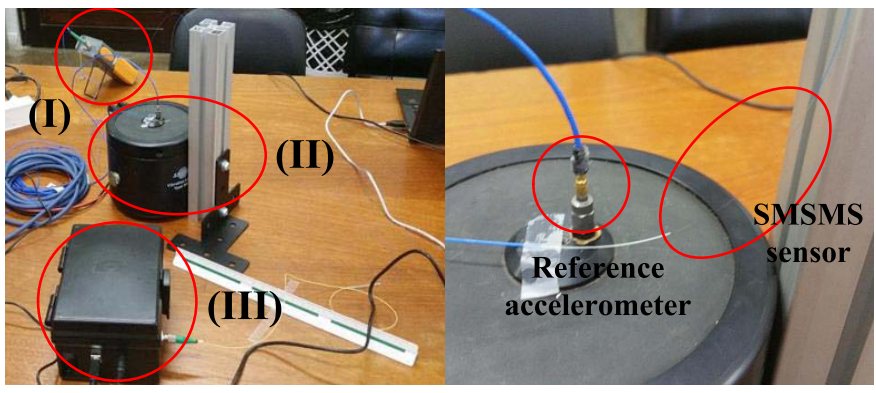

(a)

(b)

Fig. 5. Experimental setup for the SMSMS sensor. (a.I) Laser source. (a.II) Shaker with the reference accelerometer and the SMSMS sensor. (a.III) Optical DAQ system based on Arduino. (b) Zoomed-in view of a.II with the reference accelerometer and the SMSMS sensor fixed in the shaker.

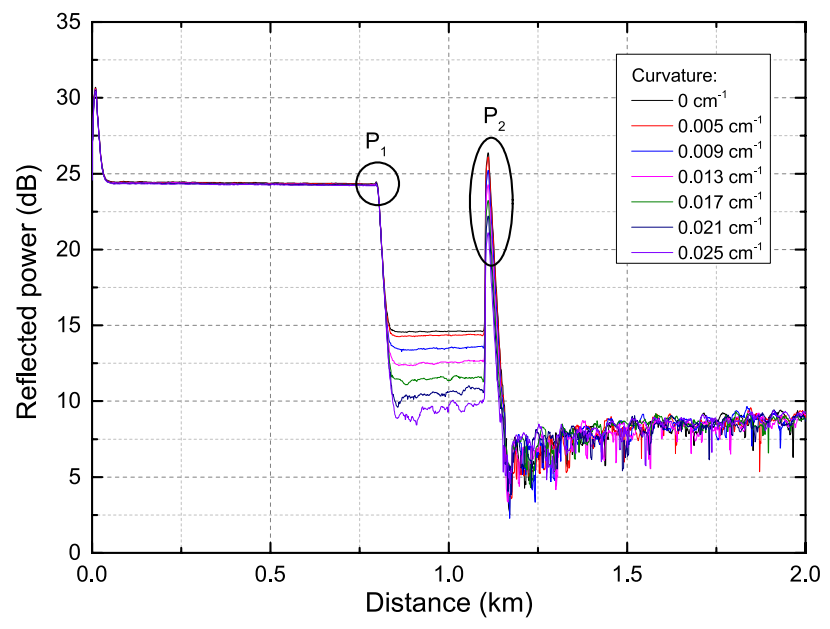

Fig. 6. OTDR trace at $1550 \mathrm{~nm}$ for the SMS 1 curvature sensor.

vibration exciter, namely, shaker. The shaker is excited by a sine signal at selected frequencies covering a low frequency range, such as $0.1,0.2,0.4,0.8,1.0$, and $10 \mathrm{~Hz}$. The optical acquisition system based on Arduino presents the following configuration: measurement data are acquired by an A/D converter, with a signal period of $\approx 5.194 \mathrm{~ms} / \mathrm{sample}$, with a gain of 11 [dimensionless parameter (volt/volt) configured in the acquisition software].

\section{RESULTS AND DISCUSSION}

In this section, experimental results, using the described setups and the fabricated sensors (SMS and SMSMS), are discussed. Besides, the experimental results from the SMS sensor are compared with numerical simulations using BPM.

\section{A. Curvature Analysis of the SMS Sensor}

To evaluate the SMS structure as a curvature sensor, a difference between optical power levels is analyzed, which represents the induced loss by the applied curvature. In the measured data, the signal power is determined as the difference between the high reflection peak value $\left(P_{2}\right)$, and the value before the loss $\left(P_{1}\right)$, as shown in Fig. 6. Thus, the data analysis is obtained with the power difference defined as $P_{\text {Dif }}=P_{2}-P_{1}$. 


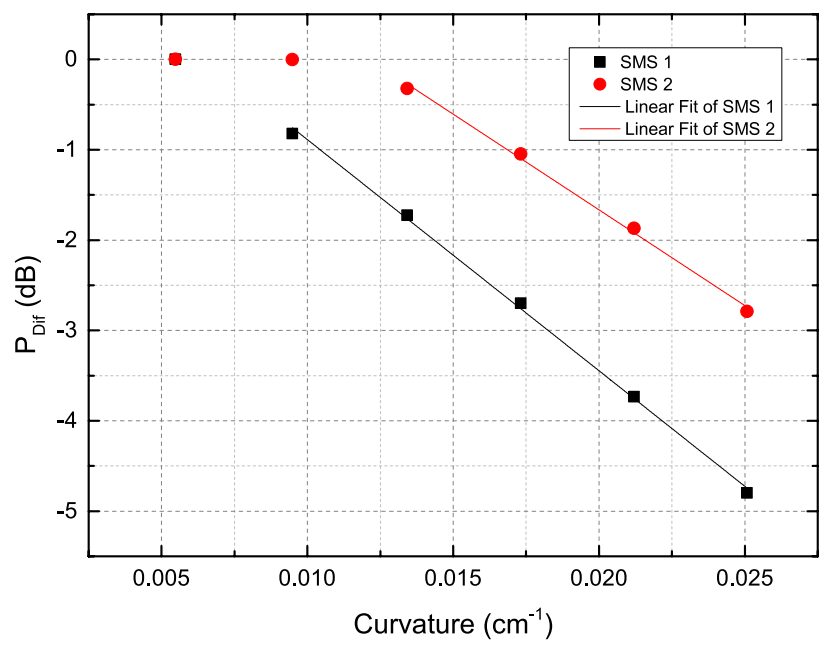

Fig. 7. $P_{\text {Dif }}$ as a function of curvature for the two SMS sensors.

In Fig. 6, the trace of the OTDR measurement to the SMS 1 sensor for six different values of curvature, from $C=0.005 \mathrm{~cm}^{-1}$ to $C=0.025 \mathrm{~cm}^{-1}$, obtained with the applied displacements, is exhibited. The OTDR trace presents the reflected optical power as a function of the distance from the OTDR to the optical fiber mirror.

After subjecting the SMS sensor to displacement, the optical losses accumulated in the full range tested are $\approx 4.9 \mathrm{~dB}$. The peak signal $P_{2}$ is due to the reflection of the deposited optical fiber mirror at the output of the $300 \mathrm{~m}$ of fiber. When the loss of SMS sensor is small, a lot of light is reflected, then $P_{2}$ is greater than $P_{1}$. On the other hand, if the bent is applied, $P_{2}$ is significantly reduced when compared with the case without curvature, due to the increase of optical losses; the arriving signal to the optical fiber mirror is reduced, coming back only $25 \%$ of light, then $P_{2}$ is smaller than $P_{1} . P_{\text {Dif }}$ for two SMS sensors as a function of the applied curvature is shown in Fig. 7.

In the curvature range from $C=0.013 \mathrm{~cm}^{-1}$ to $C=0.025 \mathrm{~cm}^{-1}$, the sensors present satisfactory sensitivity. SMS 1 presented a slope of $\approx-255.8 \mathrm{~dB} / \mathrm{cm}^{-1}$ and a linearity of $R^{2}=0.99833$. SMS 2 presented a slope of $\approx-211.8 \mathrm{~dB} / \mathrm{cm}^{-1}$ and a linearity of $R^{2}=0.99567$. Slope as a first-order approximation to estimate the sensitivity of the sensors is used.

Among these results, SMS 1 presents the sensitivity of $17.2 \%$ better than SMS 2, as shown in Table I. Nevertheless, for sensor multiplexing, the SMS 1 sensor does not exhibit the best performance, since it introduces high power loss $\approx 4.9 \mathrm{~dB}$. Through the performed experiment, one can verify that by controlling the attenuation of the sensor, it is possible to control its performance against curvature. The higher the attenuation introduced by the CDM, the higher will be the sensitivity, however depending on the dynamic range of the OTDR. Thus, one may have a set of multiplexed sensors with lower sensitivity or a single punctual sensor with higher attenuation, nevertheless with best performance, depending on the application. Note that the difference between SMS 1 and SMS 2 derived from the manufacture process.
TABLE I

CHARACTERISTICS OF THE TWO SMS SENSORS

\begin{tabular}{l|l|l}
\hline Characteristics & SMS 1 & SMS 2 \\
\hline Sensitivity $\left(\mathrm{dB} / \mathrm{cm}^{-1}\right)$ & $\approx-255.8$ & $\approx-211.8$ \\
Resolution range $\left(\mathrm{cm}^{-1}\right)$ & $\approx 0.00074$ & $\approx 0.0015$ \\
Range in OTDR trace $(\mathrm{dB})$ & 4.9 & 2.67 \\
\hline
\end{tabular}

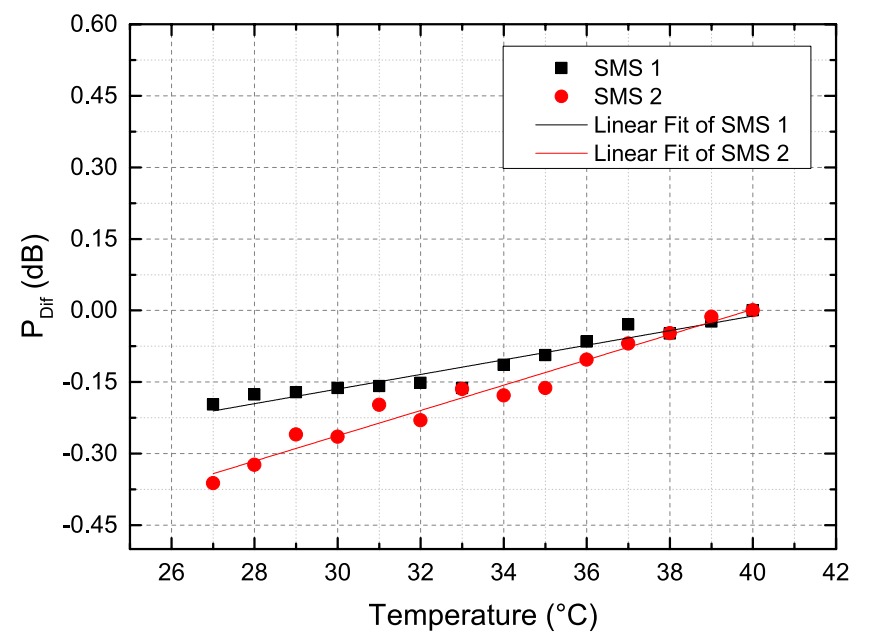

Fig. 8. $P_{\text {Dif }}$ as a function of temperature for the two SMS sensors.

\section{B. Analysis of the Temperature Influence on the SMS Sensor}

The effect of temperature in the SMS sensor can be explained by the sensitivity of the silica matrix to the temperature through its thermal expansion coefficient $(\alpha)$ and thermo-optic coefficient $(\xi)$ [50], [51]. The thermal expansion coefficient describes the physical expansion or contraction of the fiber resulting from the temperature change, and the thermo-optic coefficient describes the refractive index change of the core in response to a temperature variation. Both effects will lead small changes in the optical path of light and, consequently, slight changes in the transmitted or coupled optical power by the CDM section [52].

The SMS 1 sensor presented a slope of $\approx-0.01 \mathrm{~dB} /{ }^{\circ} \mathrm{C}$ and a linearity of $R^{2}=0.92217$, while for the SMS 2 sensor, the values attained are, respectively, $\approx-0.02 \mathrm{~dB} /{ }^{\circ} \mathrm{C}$ and $R^{2}=0.96541$. Variations in $P_{\text {Dif }}$ as a function of the applied temperature are shown in Fig. 8. The results demonstrate that the SMS 2 sensor showed a slightly different temperature sensitivity compared with the SMS 1 sensor although they exhibited similarity in their power levels. This analysis shows low sensitivity to temperature for both sensors. Thus, the influence of temperature in the curvature measurements can be considered as negligible.

\section{Vibration Analysis}

The feasibility of the SMSMS configuration is only evaluated for vibration. This configuration is not tested for curvature due to the fact that if the sensor is sensitive to vibration, it is also capable of measuring curvature.

In the performed experiment, the shaker system, type 4809, from Brüel \& Kjær is used, which has a uniform excitation 

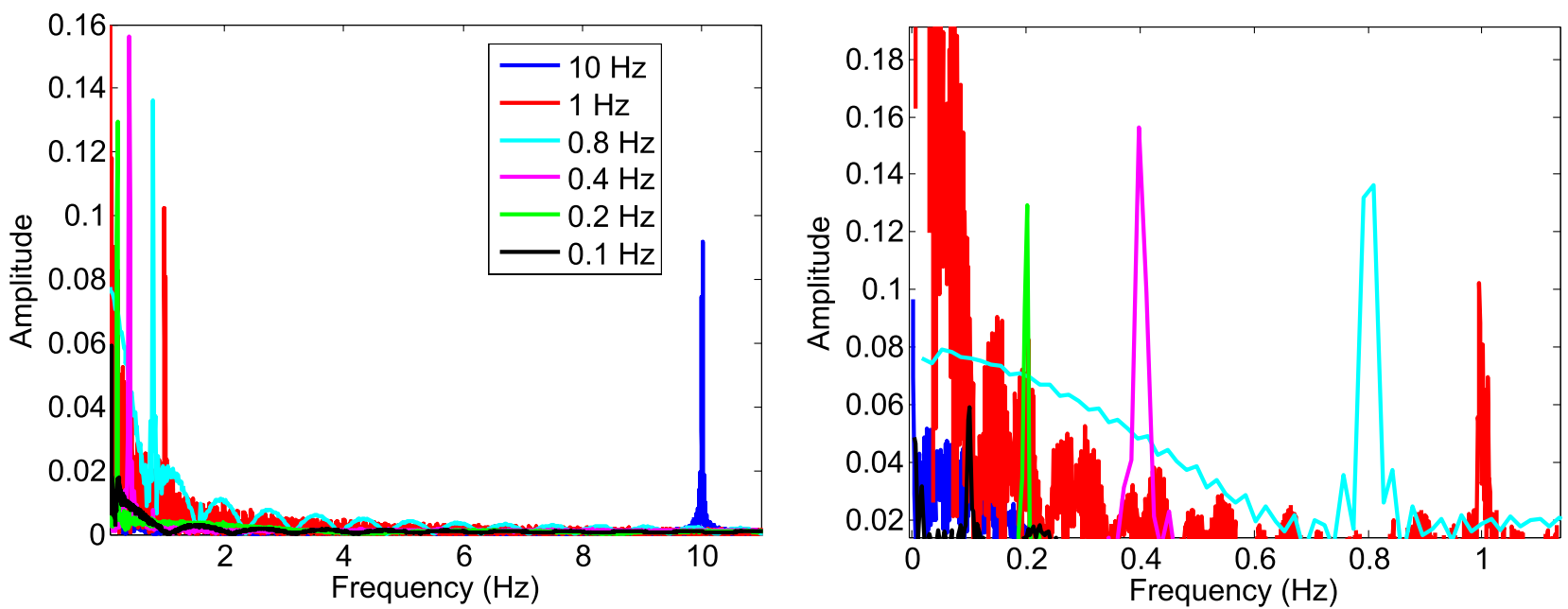

Fig. 9. Frequency spectrum of the SMSMS sensor in the range of $0.1-10 \mathrm{~Hz}$ (left) and a zoomed-in view of frequency spectrum of the SMSMS sensor in the range of $0.1-1 \mathrm{~Hz}$ (right).

in the operation range from $5 \mathrm{~Hz}$ with high stability. As an optical source, a laser source operating in the wavelength of $1550 \mathrm{~nm}$ with $-5 \mathrm{dBm}$ of optical power is used, and as the interrogation system, an optical DAQ system based on an Arduino configuration is used. Note that the fixed SMSMS sensor on the shaker is semibent to enhance the sensitivity, and the sensor captures the vibrations produced by the shaker.

The light of the laser source is injected in the SMSMS structure, and with the optical DAQ system based on an Arduino configuration, the optical power in time domain for each different frequency applied in the shaker is measured. Afterward, to obtain the spectral response of the sensor in the frequency domain, a fast Fourier transform (FFT) is applied to the signal.

When the frequencies lower than the operational range of the shaker are applied, the peaks in the frequency spectrum obtained by FFT are clearly defined, as shown in Fig. 9 (left). Thus, even with noise, the resonances at $0.1,0.2,0.4$, $0.8,1$, and $10 \mathrm{~Hz}$ are measured by the SMSMS sensor. These frequencies are excited by the shaker, but are not measured by the reference accelerometer, shown in Fig. 5(b). Fig. 9 (right) shows the frequency response of the SMSMS sensor for the range of $0.1-1 \mathrm{~Hz}$ to highlight this part of the spectrum.

In the frequency spectrum, the noises are generated by applying frequencies lower than the operation range of shaker, by environmental noise, from computer and other instruments, and thus, amplitude variations appear, as shown in Fig. 9. For instance, the resonance at $0.4 \mathrm{~Hz}$ shows a higher amplitude than 0.1 and $0.8 \mathrm{~Hz}$ resonances.

The results indicate that the proposed SMSMS as a vibration sensor has a higher sensitivity to very low frequencies in comparison with the most commercial accelerometers. This implies that the proposed SMSMS sensor can be used in frequency ranges below $1 \mathrm{~Hz}$ with a satisfactory precision to measure the low vibration frequencies of engineering structures.

\section{Numerical Simulation}

To validate the experimental response of the SMS structure as a curvature sensor, numerical simulations based
TABLE II

PARAMETERS OF THE SMS SENSOR USED IN THE NUMERICAL SimUlation

\begin{tabular}{lc|ll}
\hline SMF-28 Corning & & \\
\hline Core diameter & $8.2 \mu \mathrm{m}$ & Core refractive index & 1.4552 \\
Cladding diameter & $125 \mu \mathrm{m}$ & Cladding refractive index & 1.45 \\
\hline MMF (FG105-LCA & Thorlabs) & & \\
\hline Core diameter & $105 \mu \mathrm{m}$ & Core refractive index & 1.457 \\
Cladding diameter & $125 \mu \mathrm{m}$ & Cladding refractive index & 1.440 \\
Numerical diameter & $160 \mu \mathrm{m}$ & External refractive index & 1.0 \\
\hline
\end{tabular}

on 3-D BPM are carried out at a wavelength of $1550 \mathrm{~nm}$, using BeamPROP software from RSoft Company. The simulations apply the transverse electric (TE) polarization mode and the parameters of the SMS structure are based on the SMS sensor configuration used in the experiments, as summarized in Table II.

A nonuniform grid with maximum and minimum size in the transverse axis of 0.5 and $0.01 \mu \mathrm{m}$, respectively, is used. The grid length at the propagation axis is $0.5 \mu \mathrm{m}$ at the SMS regions and $1.5 \mu \mathrm{m}$ elsewhere. For the bend simulation, the values of the wavelength variations and the curvature radius are based on the spectral range of the OTDR and the displacement range used in the experimental analysis, respectively. The propagation of the light field through the sensor is shown in Fig. 10. The bend in the MMF section has a significant influence on the mode distribution [53]. The response of the transmitted light as a function of bend radius variations is numerically analyzed. The fundamental mode is launched at the input of the device and the bending of the device is varied. The simulations are performed through the BPM in association with a conformal mapping technique [54].

The percentage of optical power as a function of the wavelength for different curvatures is shown in Fig. 11. An average of this percentage of optical power is calculated and the simulated spectral response of the SMS sensors is obtained, as shown in Fig. 12. The normalized reflected power of the SMS sensors as a function of the bend radius is compared 

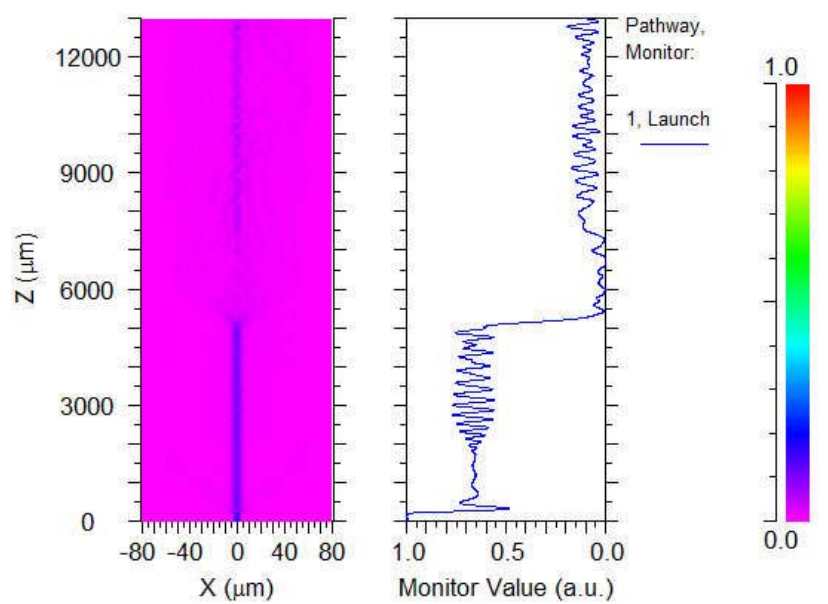

Fig. 10. Propagation of the light along the SMS sensor in 3-D BPM based on the TE polarization mode.

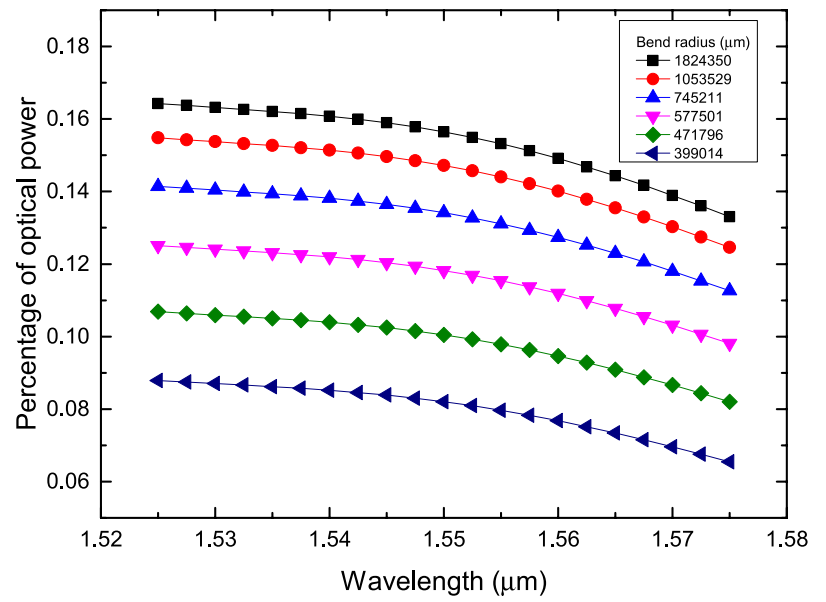

Fig. 11. Percentage of optical power as a function of the wavelength for different curvatures

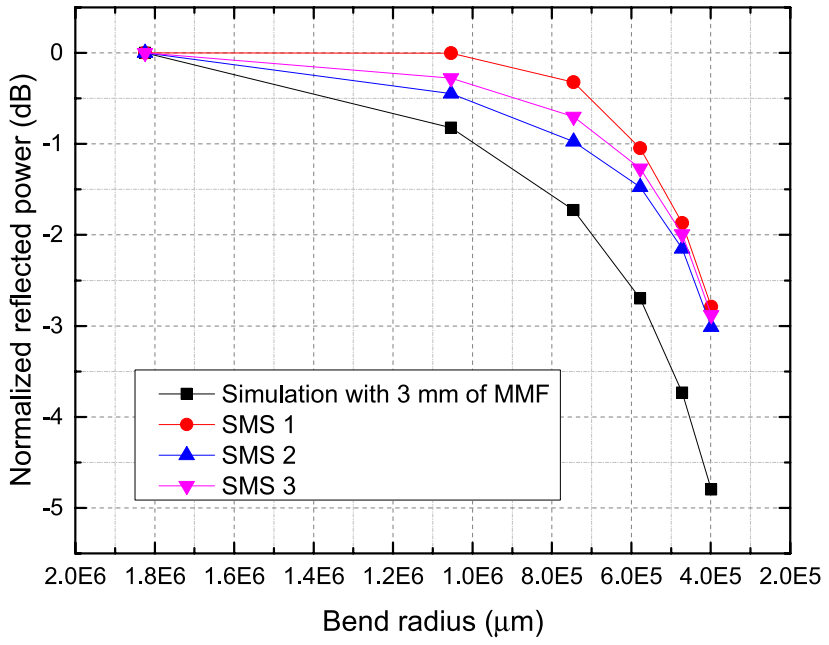

Fig. 12. Comparison between the experimental and simulated results for the SMS sensors.

with the experimental results. The calculated power variations as a function of the bend radius at a wavelength of $1550 \mathrm{~nm}$ using BeamPROP is highlighted in Fig. 12.
In addition to the two first tests, a new SMS sensor is fabricated to attest the reproducibility of the sensor in comparison with the simulated results. The curve of the simulation follows the same behavior as the curve of the experimental analysis, which confirms that the SMS structure is adequate for curvature sensing. Although the curve shows the same behavior, the simulated response is closer to the experimental results presented by the SMS 2 and SMS 3 sensors, with a difference of 0.0892 and $0.1322 \mathrm{~dB}$ for the bend radius of $4 \mathrm{E} 5$, respectively. The difference of $\approx 2.0508 \mathrm{~dB}$ from the experimental results of the SMS 1 sensor to the simulated results, SMS 2 and SMS 3 sensors, is justified due to the manufacturing process which is not very precise, thereby the sensor heads are not exactly equal, which tightly affect the sensitivity. Furthermore, the splice insertion losses can also influence the results.

\section{CONClusion}

This paper proposed the use of an SMS structure as a remote curvature sensor and an SMSMS device as a vibration sensor. The SMS sensor with an MMF length of $3 \mathrm{~mm}$ for remote curvature sensing used an OTDR interrogation system. This paper extends the feasibility of such fiber structure for curvature measurement. To increase the reflected power a fiber optical mirror was used. The results of the displacement from 0 to $600 \mu \mathrm{m}$ applied to the two SMS sensors evidenced linear behaviors with slopes of $\approx-255.8$ and $\approx-211.8 \mathrm{~dB} / \mathrm{cm}^{-1}$, respectively.

The achieved bend resolutions are $\approx 0.00074$ and $\approx 0.0015 \mathrm{~cm}^{-1}$, and a variation of 4.9 and $2.67 \mathrm{~dB}$ in the OTDR traces for the SMS 1 and SMS 2 sensors, respectively. The measurements also attested the low dependence of the SMS curvature sensor to temperature. Several sensors, such as strain, temperature, and vibration, can be used simultaneously to perform quasi-distributed curvature measurements for applications, such as SHM, depending on the dynamic range of the interrogation system only.

The SMSMS structure as a vibration sensor was used to measure vibrations in the low frequency range of 0.1-10 Hz. Experimental investigation demonstrated that this sensor is capable of detecting low frequencies, with satisfactory sensitivity and most importantly, with appropriate selection of operating frequencies and clearly defines peaks in the frequency spectrum. Compared with the conventional in-fiber accelerometer sensors, the SMSMS sensor provides a higher sensitivity in the low frequency range with a lower cost, improving the sensing robustness and simplifying the fabrication process. The proposed cost-effective device can be exploited for various sensing applications such as SHM.

\section{ACKNOWLEDGMENT}

The authors would like to thank Thorlabs Company, which kindly provided $5 \mathrm{~m}$ of MMF FG105-LCA, Prof. F. Setubal, Prof. A. Araújo, and Prof. L. Rodrigues from the Department of Mechanical Engineering of Federal University of Pará (UFPA) for the assistance with the vibration exciter equipment, and Prof. C. Rodrigues from the Department of 
Electrical Engineering of UFPA (Tucuruí) for the discussions related to the software BeamPROP simulations.

\section{REFERENCES}

[1] L. Mescia and F. Prudenzano, "Advances on optical fiber sensors," Fibers, vol. 2, no. 1, pp. 1-23, 2014.

[2] M. R. Gleeson, Y. Tomita, S. Gallego, and R. McLeod, "Advances in novel optical materials and devices," Phys. Res. Int., vol. 2013, Mar. 2013, Art. no. 379041, doi: 10.1155/2013/430947.

[3] C. K. Y. Leung et al., "Review: Optical fiber sensors for civil engineering applications," Mater. Struct., vol. 48, no. 4, pp. 871-906, Apr. 2015.

[4] X. W. Ye, Y. H. Su, and J. P. Han, "Structural health monitoring of civil infrastructure using optical fiber sensing technology: A comprehensive review," Sci. World J., vol. 2014, Jul. 2014, Art. no. 652329.

[5] K. Worden and J. M. Dulieu-Barton, "An overview of intelligent fault detection in systems and structures," Struct. Health Monitor., vol. 3, no. 1, pp. 85-98, 2004.

[6] C. R. Farrar and K. Worden, "An introduction to structural health monitoring," Philos. Trans. Roy. Soc. London A, Math. Phys. Sci., vol. 365 , no. 1851 , pp. 303-315, 2007.

[7] J. M. López-Higuera, L. Rodriguez-Cobo, A. Q. Incera, and A. Cobo, "Fiber optic sensors in structural health monitoring," J. Lightw. Technol., vol. 29, no. 4, pp. 587-608, Feb. 15, 2011

[8] W. Fan and P. Qiao, "Vibration-based damage identification methods: A review and comparative study," Struct. Health Monitor., vol. 10, no. 1, pp. 83-111, 2010.

[9] R. Wang et al., "Highly sensitive curvature sensor using an infiber Mach-Zehnder interferometer," IEEE Sensors J., vol. 13, no. 5, pp. 1766-1770, May 2013

[10] A. Djordjevich and Y. He, "Curvature measurements," Proc. SPIE, vol. 3555, pp. 322-329, Aug. 1998, doi: 10.1117/12.318211.

[11] D. Goyal and B. S. Pabla, "The vibration monitoring methods and signal processing techniques for structural health monitoring: A review," Arch. Comput. Methods Eng., pp. 1-10, Mar. 2015, doi: 10.1007/s11831-0159145-0.

[12] A. Djordjevich, "Alternative to strain measurement," Opt. Eng., vol. 42, no. 7, pp. 1888-1892, Jul. 2003.

[13] X. Yan, W. Huang, S. R. Kwon, S. Yang, X. Jiang, and F.-G. Yuan, "A sensor for the direct measurement of curvature based on flexoelectricity," Smart Mater. Struct., vol. 22, no. 8, p. 085016, Jul. 2013.

[14] A. Djordjevich and Y. He, "Thin structure deflection measurement," IEEE Trans. Instrum. Meas., vol. 48, no. 3, pp. 705-710, Jun. 1999.

[15] D. Inaudi, S. Vurpillot, N. Casanova, and P. Kronenberg, "Structural monitoring by curvature analysis using interferometric fiber optic sensors," Smart Mater. Struct., vol. 7, no. 2, pp. 199-208, 1998.

[16] P. F. da Costa Antunes et al., "Optical fiber accelerometer system for structural dynamic monitoring," IEEE Sensors J., vol. 9, no. 11, pp. 1347-1354, Nov. 2009.

[17] G. Rajan et al., "Analysis of vibration measurements in a composite material using an embedded PM-PCF polarimetric sensor and an FBG sensor," IEEE Sensors J., vol. 12, no. 5, pp. 1365-1371, May 2012.

[18] M. Çelebi, "Seismic monitoring of structures and new developments," in Earthquakes and Health Monitoring of Civil Structures (Springer Environmental Science and Engineering), M. Garevski, Ed. Dordrecht, The Netherlands: Springer, 2013, pp. 37-84.

[19] Y. R. García, J. M. Corres, and J. Goicoechea, "Vibration detection using optical fiber sensors," J. Sensors, vol. 2010, Jul. 2010, Art. no. 936487.

[20] P. Moyo, J. M. W. Brownjohn, R. Suresh, and S. C. Tjin, "Development of fiber Bragg grating sensors for monitoring civil infrastructure," Eng. Struct., vol. 27, no. 12, pp. 1828-1834, Oct. 2005.

[21] Y.-P. Wang and Y.-J. Rao, "A novel long period fiber grating sensor measuring curvature and determining bend-direction simultaneously," IEEE Sensors J., vol. 5, no. 5, pp. 839-843, Oct. 2005.

[22] J.-O. Gaudron, F. Surre, T. Sun, and K. T. V. Grattan, "Long period grating-based optical fibre sensor for the underwater detection of acoustic waves," Sens. Actuators A, Phys., vol. 201, pp. 289-293, Oct. 2013.

[23] P. A. R. Tafulo, P. A. S. Jorge, J. L. Santos, F. M. Araujo, and O. Frazao, "Intrinsic Fabry-Pérot cavity sensor based on etched multimode graded index fiber for strain and temperature measurement," IEEE Sensors J., vol. 12 , no. 1 , pp. 8-12, Jan. 2012.

[24] A. Kersey et al., "Fiber grating sensors," J. Lightw. Technol., vol. 15 , no. 8, pp. 1442-1463, Aug. 1997.

[25] O. Frazão, L. A. Ferreira, F. M. Araújo, and J. L. Santos, "Applications of fiber optic grating technology to multi-parameter measurement," Fiber Integr. Opt., vol. 24, nos. 3-4, pp. 227-244, 2005.
[26] Y.-J. Rao, "In-fibre Bragg grating sensors," Meas. Sci. Technol., vol. 8, no. 4, pp. 355-375, 1997

[27] J. Salo and I. Korhonen, "Calculated estimate of FBG sensor's suitability for beam vibration and strain measuring," Measurement, vol. 47, pp. 178-183, Jan. 2014.

[28] Z.-S. Guo, "Strain and temperature monitoring of asymmetric composite laminate using FBG hybrid sensors," Struct. Health Monitor., vol. 6, no. 3, pp. 191-197, Sep. 2007.

[29] P. Antunes, H. Varum, and P. André, "Uniaxial fiber Bragg grating accelerometer system with temperature and cross axis insensitivity," Measurement, vol. 44, no. 1, pp. 55-59, Jan. 2011.

[30] N. Basumallick, I. Chatterjee, P. Biswas, K. Dasgupta, and S. Bandyopadhyay, "Fiber Bragg grating accelerometer with enhanced sensitivity," Sens. Actuators A, Phys., vol. 173, no. 1, pp. 108-115, 2012.

[31] A. Laudati et al., "A fiber optic Bragg grating seismic sensor," Proc. SPIE, vol. 6619, pp. 66191C-1-66191C-4, Jul. 2007.

[32] M. S. Müller et al., "Fiber-optic sensor interrogation based on a widely tunable monolithic laser diode," IEEE Trans. Instrum. Meas., vol. 59 , no. 3, pp. 696-703, Mar. 2010

[33] A. Vallan, A. Carullo, M. L. Casalicchio, and G. Perrone, "Design and characterization of curvature sensors based on plastic optical fibers for structural monitoring," in Proc. IEEE Int. Instrum. Meas. Technol. Conf. (I2MTC), May 2013, pp. 996-1000.

[34] H. Fu et al., "High-sensitivity Mach-Zehnder interferometric curvature fiber sensor based on thin-core fiber," IEEE Sensors J., vol. 15, no. 1 , pp. 520-525, Jan. 2015.

[35] A. E. A. Hatta, Y. Semenova, G. Rajan, and G. Farrell, "A voltage sensor based on a singlemode-multimode-singlemode fiber structure," Microw. Opt. Technol. Lett., vol. 52, no. 8, pp. 1887-1890, Aug. 2010.

[36] Y. Gong, T. Zhao, Y.-J. Rao, and Y. Wu, "All-fiber curvature sensor based on multimode interference," IEEE Photon. Technol. Lett., vol. 23, no. 11, pp. 679-681, Jun. 1, 2011

[37] Q. Wu, A. M. Hatta, P. Wang, Y. Semenova, and G. Farrell, "Use of a bent single SMS fiber structure for simultaneous measurement of displacement and temperature sensing," IEEE Photon. Technol. Lett., vol. 23 , no. 2, pp. 130-132, Jan. 15, 2011

[38] A. M. Hatta, K. Indriawati, T. Bestariyan, T. Humada, and S. Koentjoro, "SMS fiber structure for temperature measurement using an OTDR," Photon. Sensors, vol. 3, no. 3, pp. 262-266, 2013.

[39] A. M. Hatta, H. E. Permana, H. Setijono, A. Kusumawardhani, and S. Koentjoro, "Strain measurement based on SMS fiber structure sensor and OTDR," Microw. Opt. Technol. Lett., vol. 55, no. 11, pp. 2576-2578, Nov. 2013.

[40] Y. Zhao, X.-G. Li, F.-C. Meng, and Z. Zhao, "A vibration-sensing system based on SMS fiber structure," Sens. Actuators A, Phys., vol. 214 pp. 163-167, Aug. 2014.

[41] M. T. M. R. Giraldi et al., "Fiber optic displacement sensor based on a double-reflecting OTDR technique," Microw. Opt. Technol. Lett., vol. 57, no. 6, pp. 1312-1315, Jun. 2015.

[42] A. Arifin, A. Hatta, S. Koentjoro, M. S. Muntini, and A. Rubiyanto, "Long-range displacement sensor based on SMS fiber structure and OTDR," Photon. Sensors, vol. 5, no. 2, pp. 166-171, Jun. 2015.

[43] J. Villatoro and D. Monzón-Hernández, "Low-cost optical fiber refractive-index sensor based on core diameter mismatch," J. Lightw. Technol., vol. 24, no. 3, pp. 1409-1413, Mar. 2006

[44] D. Donlagic and B. Culshaw, "Propagation of the fundamental mode in curved graded index multimode fiber and its application in sensor systems," J. Lightw. Technol., vol. 18, no. 3, pp. 334-342, Mar. 2000.

[45] S. Silva et al., "Curvature and temperature discrimination using multimode interference fiber optic structures-A proof of concept," J. Lightw. Technol., vol. 30, no. 23, pp. 3569-3575, Dec. 1, 2012

[46] X. Fang, C. R. Liao, and D. N. Wang, "Femtosecond laser fabricated fiber Bragg grating in microfiber for refractive index sensing," Opt. Lett., vol. 35, no. 7, pp. 1007-1009, Apr. 2010.

[47] R. Xiong et al., "Simultaneous measurement of refractive index and temperature based on modal interference," IEEE Sensors J., vol. 14, no. 8, pp. 2524-2528, Aug. 2014.

[48] Q. Rong, X. Qiao, R. Wang, H. Sun, M. Hu, and Z. Feng, "Highsensitive fiber-optic refractometer based on a core-diameter-mismatch Mach-Zehnder interferometer," IEEE Sensors J., vol. 12, no. 7, pp. 2501-2505, Jul. 2012.

[49] C. Caucheteur, K. Chah, F. Lhomme, M. Blondel, and P. Megret, "Simultaneous bend and temperature sensor using tilted FBG," Proc. SPIE, vol. 5855, pp. 707-710, Aug. 2005.

[50] E. Li, "Temperature compensation of multimode-interference-based fiber devices," Opt. Lett., vol. 32, no. 14, pp. 2064-2066, Jul. 2007. 
[51] R. Fuentes-Fuentes, D. A. May-Arrioja, J. R. Guzman-Sepulveda, M. Torres-Cisneros, and J. J. Sánchez-Mondragón, "Highly sensitive liquid core temperature sensor based on multimode interference effects," Sensors, vol. 15, no. 10, pp. 26929-26939, Oct. 2015.

[52] J. G. Aguilar-Soto, J. E. Antonio-Lopez, J. J. Sanchez-Mondragon, and D. A. May-Arrioja, "Fiber optic temperature sensor based on multimode interference effects," J. Phys., Conf. Ser., vol. 274, no. 1, p. 012011, 2011.

[53] Q. Wu et al., "The use of a bend singlemode-multimodesinglemode (SMS) fibre structure for vibration sensing," Opt. Laser Technol., vol. 63, pp. 29-33, Nov. 2014.

[54] R. Baets and P. E. Lagasse, "Loss calculation and design of arbitrarily curved integrated-optic waveguides," J. Opt. Soc. Amer., vol. 73, no. 2, pp. 177-182, Feb. 1983.

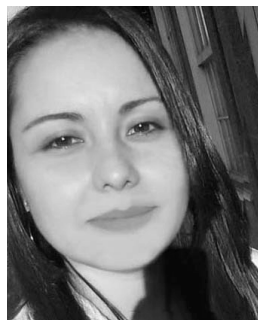

Cindy S. Fernandes received the B.Sc. degree in telecommunications engineering from the Institute of Advanced Studies of the Amazon, Belém, Brazil, in 2005 , and the M.Sc. degree in mechanical engineering and the Ph.D. degree in electrical engineering from the Federal University of Pará (UFPA), Belém, in 2011 and 2016, respectively.

She has been a member of the Applied Electromagnetism Laboratory with UFPA since 2012. Her current research interests include the optical communication and sensors, more specifically with optical devices, systems, and fiber sensors.

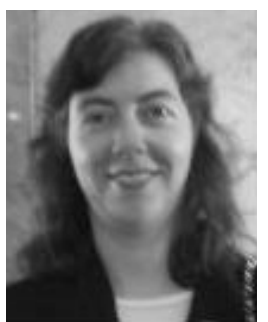

Maria Thereza M. Rocco Giraldi received the B.Sc. degree in communications engineering from Federal Fluminense University, Niterói, Brazil, in 1986, the M.Sc. degree in electrical engineering from the Pontifical Catholic University of Rio de Janeiro, Rio de Janeiro, Brazil, in 1989, and the $\mathrm{Ph} . \mathrm{D}$. degree in electrical engineering from the University of Campinas, Campinas, Brazil, in 1995.

She is currently an Associate Professor with the Military Institute of Engineering, Brazilian Army, Rio de Janeiro. She has been involved in optical communication and sensors, more specifically with optical devices and systems, Wavelength-division multiplexing systems, free-space optics communications, optical amplifiers, and fiber sensors.

Prof. Giraldi is the Editor-in-Chief of the Journal of Microwaves, Optoelectronics and Electromagnetic Applications, a journal published by the Brazilian Microwave and Optoelectronics Society and the Brazilian Electromagnetics Society.

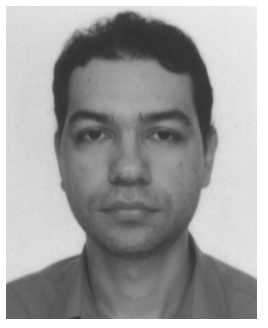

embedded systems.

Marco J. de Sousa received the M.Sc. and $\mathrm{Ph} . \mathrm{D}$. degrees in electrical engineering from the Federal University of Pará (UFPA), Belém, Brazil, in 2003 and 2008, respectively.

He has been a member of the Applied Electromagnetism Laboratory with UFPA since 1999. He joined as an Assistant Professor with UFPA in 2009, where he is currently involved in the area of sensors and embedded systems. His current research interests include optimization, optical communications, optical sensors systems, optoelectronic devices, and

Dr. de Sousa received the Title of Electrical Engineer from UFPA in 2002.

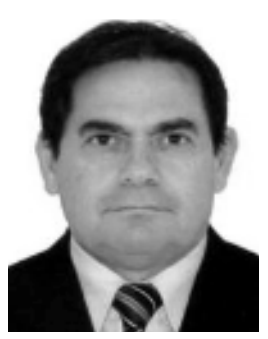

João C. W. A. Costa (S'94-M'95) received the B.Sc. degree from the Federal University of Pará (UFPA), Belém, Brazil, in 1981, the M.Sc. degree from the Pontifical Catholic University of Rio de Janeiro, Rio de Janeiro, Brazil, in 1989, and the Ph.D. degree from the University of Campinas, Campinas, Brazil, in 1994, all in electrical engineering.

He was the Deputy Secretary of Development, Science and Technology of Pará from 2007 to 2010. From 2010 to 2012, he was the Vice President of the Brazilian Microwave and Optoelectronics Society. From 2013 to 2016, he was the Vice Rector of the Federal University of Southern and Southeastern Pará, Marabá, Brazil. He is currently a Professor with the Institute of Technology, UFPA, and a Researcher with the National Council for Scientific and Technological Development, Brasília, Brazil, a Brazilian research funding agency. His current research interests include broadband systems and optical sensors.

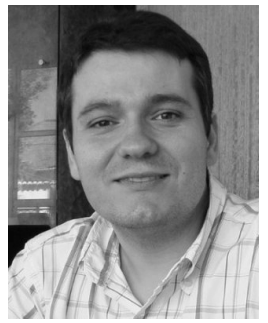

Carlos Gouveia received the B.Sc. and M.Sc. degrees in telecommunications and networks engineering and the Ph.D. degree in electrical engineering from the University of Madeira, Funchal, Portugal, in 2007, 2008, and 2014, respectively.

$\mathrm{He}$ was with the Optoelectronics Unit, Institute for Systems and Computer Engineering of Porto (INESC Porto), Porto, Portugal, from 2008 to 2014. The research activities were hosted by INESC Porto, supported by several research and development projects and he was involved in several fundamental and technological aspects of optical fiber sensors. During the Ph.D. degree, he was also an Invited Researcher in several international universities, such as the University of Campinas, Campinas, Brazil, the University of Lund, Lund, Sweden, and the University of Pécs, Pécs, Hungary. Since 2014, he has been a Post-Doctoral Researcher of INESC P\&D Brazil by the Federal University of Campina Grande (UFCG) in the Electrical Engineering Area. His current research interests include optical fiber devices for sensing and communications, high voltage system instrumentation, signal processing, and electronics.

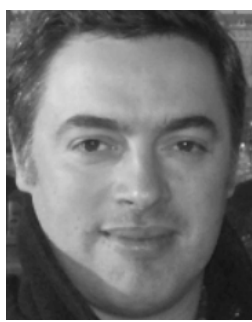

Pedro Jorge received the B.Sc. degree in applied physics (optics and lasers) from the University of Minho, Braga, Portugal, in 1996, the M.Sc. degree in optoelectronics and lasers from the Physics Department, University of Porto, Porto, Portugal, in 2000, and the Ph.D. degree from the University of Porto, in collaboration with the Department of Physics and Optical Sciences, University of Charlotte, Charlotte, NC, USA, in 2006.

He is currently a Senior Researcher with the Instituto de Engenharia de Sistemas e Computadores, Porto, where he leads the Biochemical Sensors Team exploring the potential of optical fiber and integrated optics technologies in environmental and medical applications and coordinating several projects in the area. He has published more than 150 publications in the field of sensors in national and international conferences and peer-reviewed journals. He has authored two book chapters and also holds one patent.

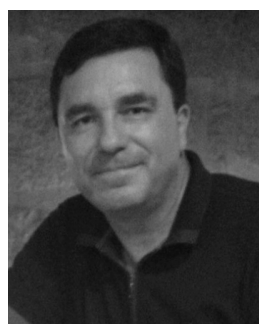

Marcos A. R. Franco received the B.S. degree in physics from the Pontifical Catholic University of Rio de Janeiro, Rio de Janeiro, Brazil, in 1983, and the M.S. degree in physics and the Ph.D. degree in electrical engineering from the University of São Paulo, São Paulo, Brazil, in 1991 and 1999, respectively.

$\mathrm{He}$ is currently a Researcher in the postgraduate program in science and space technologies with the Institute of Advanced Studies (IEAv), São José dos Campos, Brazil, and a Professor with the Aeronautics Institute of Technology. He is the Head of the Computational Electromagnetic Laboratory with IEAv. His current research interests include the applied electromagnetism computational modeling of electromagnetic devices, optical fibers, fiber optic sensors, photonic crystal fibers, microstructured optical fibers, and terahertz devices. 